\title{
QUANTUM ERGODIC RESTRICTION FOR CAUCHY DATA: INTERIOR QUE AND RESTRICTED QUE
}

\author{
Hans Christianson, John A. Toth and Steve Zelditch
}

\begin{abstract}
We prove a quantum ergodic restriction theorem for the Cauchy data of a sequence of quantum ergodic eigenfunctions on a hypersurface $H$ of a Riemannian manifold $(M, g)$. The technique of proof is to use a Rellich-type identity to relate quantum ergodicity of Cauchy data on $H$ to quantum ergodicity of eigenfunctions on the global manifold $M$. This has the interesting consequence that if the eigenfunctions are quantum uniquely ergodic on the global manifold $M$, then the Cauchy data is automatically quantum uniquely ergodic on $H$ with respect to operators whose symbols vanish to order one on the glancing set of unit tangential directions to $H$.
\end{abstract}

\section{Introduction}

This paper is concerned with the quantum ergodic restriction (QER) problem for hypersurfaces in compact Riemannian manifolds $(M, g)$. We consider the eigenvalue problem on $M$

$$
\left\{\begin{array}{l}
-\Delta_{g} \varphi_{j}=\lambda_{j}^{2} \varphi_{j}, \quad\left\langle\varphi_{j}, \varphi_{k}\right\rangle=\delta_{j k} \\
B \varphi_{j}=0 \text { on } \partial M
\end{array},\right.
$$

where $\langle f, g\rangle=\int_{M} f \bar{g} d V$ ( $d V$ is the volume form of the metric) and where $B$ is the boundary operator, e.g., $B \varphi=\left.\varphi\right|_{\partial M}$ in the Dirichlet case or $B \varphi=\left.\partial_{\nu} \varphi\right|_{\partial M}$ in the Neumann case. We also allow $\partial M=\emptyset$. We introduce the Planck constant $h_{j}=\lambda_{j}^{-1}$; for notational simplicity we often drop the subscript $j$. We then denote the eigenfunctions in the orthonormal basis by $\varphi_{h}$ and the eigenvalues by $h^{-2}$, so that the eigenvalue problem takes the semi-classical form,

$$
\left\{\begin{array}{l}
\left(-h^{2} \Delta_{g}-1\right) \varphi_{h}=0 \\
B \varphi_{h}=0 \text { on } \partial M
\end{array}\right.
$$

where $B=I$ or $B=h D_{\nu}$ in the Dirichlet or Neumann cases respectively. Also, $\Delta_{g}$ denotes the negative Laplacian, e.g., on flat $\mathbb{R}^{n}$ it denotes $\sum_{j=1}^{n} \frac{\partial^{2}}{\partial x_{j}^{2}}$.

Let $H \subset M$ be a smooth hypersurface which does not meet $\partial M$ if $\partial M \neq \emptyset$. The main result of this paper (Theorem 1 ) is that the semiclassical Cauchy data

$$
C D\left(\varphi_{h}\right):=\left\{\left(\left.\varphi_{h}\right|_{H},\left.h D_{\nu} \varphi_{h}\right|_{H}\right)\right\}
$$

Received by the editors May 8, 2012. 
of eigenfunctions is always quantum ergodic along any hypersurface $H \subset M$ if the eigenfunctions are quantum ergodic on the global manifold $M$. The proof is a generalization of the boundary case where $H=\partial M$, which was proved in [HaZe] and in [Bu]. Our proof is modelled on that of [Bu], developing ideas of [GL]. This automatic QER property of Cauchy data stands in contrast to the conditional nature of the QER property for the Dirichlet data alone, which requires an "asymmetry" condition on $H$ with respect to geodesics [TZ1, TZ2, DZ]. Note that in the boundary case $H=\partial M$, the Dirichlet resp. Neumann boundary condition kills one of the two components of the Cauchy data, so that the Cauchy data QER theorem appears the same as the QER theorem for Neumann data (resp. Dirichlet data) alone.

As emphasized below the statement of Theorem 1, the deduction of quantum ergodicity of the Cauchy data from quantum ergodicity of the sequence of ambient eigenfunctions holds for the full original sequence. Hence, if the original sequence is a complete orthonormal basis of eigenfunctions, i.e., if $\Delta_{g}$ is quantum uniquely ergodic (QUE), then the Cauchy data of the full orthonormal basis is also QUE on $H$ for any embedded orientable separating hypersurface $H$. We refer to this as the QUER property. However, it is not necessarily the case that the Cauchy data are QUER for the full algebra of pseudodifferential operators. In Corollary 1.1, it is proved that QUE on $(M, g)$ implies QUER with respect to the subalgebra of semiclassical pseudodifferential operators on $H$ whose symbols vanish to order 1 along $S^{*} H$. The restriction on the symbols arises because the passage from QUE in the ambient manifold to QUER on the hypersurface involves multiplying the symbols by a certain factor which vanishes to order one on $S^{*} H$, i.e., the unit directions (co-)tangent to $H$. Therefore, QUE in the ambient manifold does not imply QUER for all pseudodifferential operators on $H$, and indeed the test operators damp out the possible modes which concentrate microlocally on $T^{*} H$. We nevertheless refer to it as a QUER property because it holds for the entire sequence of eigenfunctions; there is no need to remove a subsequence of density zero for the subalgebra limits.

To state the results precisely, we introduce some notation. We work with the semiclassical calculus of pseud-differential operators as in the references [Bu, DZ, HaZe, TZ1, Zw]; see also Appendix A for background. On both $H$ and $M$ we fix (Weyl) quantizations $a \rightarrow a^{w}$ of semi-classical symbols to semi-classical pseudodifferential operators. When it is necessary to indicate which manifold is involved, we either write $O p_{H}(a)$ for pseudodifferential operators on $H$ or we use capital letters $A^{w}(x, h D)$ to indicate operators on $M$ and small letters $a^{w}\left(y, h D_{y}\right)$ to indicate operators on $H$.

A sequence of functions $u_{h_{j}}$ on a manifold $M$ indexed by a sequence of Planck constants is said to be quantum ergodic with limit measure $d \mu$ if

$$
\left\langle a^{w}\left(x, h_{j} D_{x}\right) u_{j}, u_{j}\right\rangle \rightarrow \omega\left(a_{0}\right):=\int_{T^{*} M} a_{0} d \mu
$$

for all zeroth order semi-classical pseudodifferential operators, where $a_{0}$ is the principal symbol of $a^{w}\left(x, h_{j} D_{x}\right)$. In the classical case of compact Riemannian manifolds $(M, g)$ with ergodic geodesic flow of [Sch, Zel, CdV, ZZ], the $\left\{u_{j}\right\}$ were a subsequence of density one of an orthonormal basis of eigenfunctions of $\Delta_{g}$ and $d \mu$ is normalized Liouville measure on $S^{*} M$. The functional $a \rightarrow\left\langle a^{w}\left(x, h_{j} D_{x}\right) u_{j}, u_{j}\right\rangle$ is often referred to as a microlocal lift (or a Wigner distribution), and the limit measure or state $\omega(a)$ is often called a quantum limit or a semi-classical defect measure. Thus, we use the 
term 'quantum ergodic sequence' in this paper to mean a sequence of functions whose microlocal lifts have a unique weak* limit. For the results of this paper, it is not necessary to assume that the geodesic flow is ergodic; we show that that Cauchy data of a quantum ergodic sequence of eigenfunctions in the ambient space $M$ are quantum ergodic on $H$, no matter what mechanism leads to quantum ergodicity of the original sequence.

We introduce a hypersurface $H$, which we assume to be orientable, embedded, and separating in the sense that

$$
M \backslash H=M_{+} \cup M_{-}
$$

where $M_{ \pm}$are domains with boundary in $M$. This is not a restrictive assumption since we can arrange that any hypersurface is a separating hypersurface.

Given a quantization $a \rightarrow O p_{H}(a)$ of semi-classical symbols $a \in S_{s c}^{0}(H)$ of order zero (see Appendix A) to semi-classical pseudodifferential operators on $L^{2}(H)$, we define the microlocal lifts of the Neumann data as the linear functionals on $a \in S_{s c}^{0}(H)$ given by

$$
\mu_{h}^{N}(a):=\int_{B^{*} H} a d \Phi_{h}^{N}:=\left\langle\left. O p_{H}(a) h D_{\nu} \varphi_{h}\right|_{H},\left.h D_{\nu} \varphi_{h}\right|_{H}\right\rangle_{L^{2}(H)} .
$$

We also define the renormalized microlocal lifts of the Dirichlet data by

$$
\mu_{h}^{D}(a):=\int_{B^{*} H} a d \Phi_{h}^{R D}:=\left\langle\left. O p_{H}(a)\left(1+h^{2} \Delta_{H}\right) \varphi_{h}\right|_{H},\left.\varphi_{h}\right|_{H}\right\rangle_{L^{2}(H)} .
$$

Finally, we define the microlocal lift $d \Phi_{h}^{C D}$ of the Cauchy data to be the sum

$$
d \Phi_{h}^{C D}:=d \Phi_{h}^{N}+d \Phi_{h}^{R D} .
$$

Here, $h^{2} \Delta_{H}$ denotes the negative tangential Laplacian for the induced metric on $H$, so that the operator $\left(1+h^{2} \Delta_{H}\right)$ is characteristic precisely on the glancing set $S^{*} H$ of $H$. Intuitively, we have renormalized the Dirichlet data by damping out the whispering gallery components.

The distributions $\mu_{h}^{N}, \mu_{h}^{D}$ are asymptotically positive, but are not normalized to have mass one and may tend to infinity. They depend on the choice of quantization, but their possible weak* limits as $h \rightarrow 0$ do not, and the results of the paper are valid for any choice of quantization. We refer to Appendix A or to $[\mathrm{Zw}]$ for background on semi-classical microlocal analysis.

Our first result is that the Cauchy data of a sequence of quantum ergodic eigenfunctions restricted to $H$ is automatically QER for semiclassical pseudodifferential operators with symbols vanishing on the glancing set $S^{*} H$, i.e., that

$$
d \Phi_{h}^{C D} \rightarrow \omega
$$

where

$$
\omega(a)=\frac{4}{\mu\left(S^{*} M\right)} \int_{B^{*} H} a_{0}\left(x^{\prime}, \xi^{\prime}\right)\left(1-\left|\xi^{\prime}\right|^{2}\right)^{1 / 2} d \sigma
$$

is the limit state of Theorem 1. This was proved in a different way in [TZ1] in the case of piecewise smooth Euclidean domains. The assumption $H \cap \partial M=\emptyset$ is for simplicity of exposition and because the case $H=\partial M$ is already known. 
Theorem 1. Suppose $H \subset M$ is a smooth, codimension 1 embedded orientable separating hypersurface and assume $H \cap \partial M=\emptyset$. Assume that $\left\{\varphi_{h}\right\}$ is a quantum ergodic sequence of eigenfunctions (1.1). Then the sequence $\left\{d \Phi_{h}^{C D}\right\}$ (1.3) of microlocal lifts of the Cauchy data of $\varphi_{h}$ is quantum ergodic on $H$ in the sense that for any $a \in S_{s c}^{0}(H)$,

$$
\begin{aligned}
& \left\langle\left. O p_{H}(a) h D_{\nu} \varphi_{h}\right|_{H},\left.h D_{\nu} \varphi_{h}\right|_{H}\right\rangle_{L^{2}(H)}+\left\langle\left. O p_{H}(a)\left(1+h^{2} \Delta_{H}\right) \varphi_{h}\right|_{H},\left.\varphi_{h}\right|_{H}\right\rangle_{L^{2}(H)} \\
& \rightarrow_{h \rightarrow 0^{+}} \frac{4}{\mu\left(S^{*} M\right)} \int_{B^{*} H} a_{0}\left(x^{\prime}, \xi^{\prime}\right)\left(1-\left|\xi^{\prime}\right|^{2}\right)^{1 / 2} d \sigma
\end{aligned}
$$

where $a_{0}\left(x^{\prime}, \xi^{\prime}\right)$ is the principal symbol of $O p_{H}(a),-h^{2} \Delta_{H}$ is the induced tangential (semiclassical) Laplacian with principal symbol $\left|\xi^{\prime}\right|^{2}, \mu$ is the Liouville measure on $S^{*} M$, and $d \sigma$ is the standard symplectic volume form on $B^{*} H$.

Remark. We emphasize that the limit along $H$ in Theorem 1 holds for the full sequence $\left\{\varphi_{h}\right\}$. Thus, if the full sequence of eigenfunctions is known to be quantum ergodic, i.e., if the sequence is QUE, then the conclusion of the theorem applies to the full sequence of eigenfunctions.

We also remark that although we do not state it formally, Theorem 1 (and indeed all the results in this paper) apply equally well to quasimodes, that is, to approximate eigenfunctions satisfying

$$
\left\|\left(-h^{2} \Delta_{g}-1\right) \varphi_{h}\right\|_{L^{2}}=o(h)\left\|\varphi_{h}\right\|_{L^{2}}
$$

as $h \rightarrow 0+$.

The proof simply relates the interior and restricted microlocal lifts and reduces the QER property along $H$ to the QE property of the ambient manifold. If we assume that QUE holds in the ambient manifold, we automatically get QUER, which is our first Corollary:

Corollary 1.1. Suppose that $\left\{\varphi_{h}\right\}$ is QUE on $M$. Then the distributions $\left\{d \Phi_{h}^{C D}\right\}$ have a unique weak* limit

$$
\omega(a):=\frac{4}{\mu\left(S^{*} M\right)} \int_{B^{*} H} a_{0}\left(x^{\prime}, \xi^{\prime}\right)\left(1-\left|\xi^{\prime}\right|^{2}\right)^{1 / 2} d \sigma .
$$

We note that $d \Phi_{h}^{C D}$ involves the microlocal lift $d \Phi_{h}^{R D}$ rather than the microlocal lift of the Dirichlet data. However, in Theorem 2, we see that the analogue of Theorem 1 holds for a density one subsequence if we use the further renormalized distributions $d \Phi_{h}^{D}+d \Phi_{h}^{R N}$ where the microlocal lift $d \Phi_{h}^{D} \in \mathcal{D}^{\prime}\left(B^{*} H\right)$ of the Dirichlet data of $\varphi_{h}$ is defined by

$$
\int_{B^{*} H} a d \Phi_{h}^{D}:=\left\langle\left. O p_{H}(a) \varphi_{h}\right|_{H},\left.\varphi_{h}\right|_{H}\right\rangle_{L^{2}(H)},
$$

and

$$
\int_{B^{*} H} a d \Phi_{h}^{R N}:=\left\langle\left.\left(1+h^{2} \Delta_{H}+i 0\right)^{-1} O p_{H}(a) h D_{\nu} \varphi_{h}\right|_{H},\left.h D_{\nu} \varphi_{h}\right|_{H}\right\rangle_{L^{2}(H)} .
$$

Theorem 2. Suppose $H \subset M$ is a smooth, codimension 1 embedded orientable separating hypersurface and assume $H \cap \partial M=\emptyset$. Assume that $\left\{\varphi_{h}\right\}$ is a quantum ergodic 
sequence. Then, there exists a sub-sequence of density one as $h \rightarrow 0^{+}$such that for all $a \in S_{s c}^{0}(H)$,

$$
\begin{aligned}
& \left\langle\left.\left(1+h^{2} \Delta_{H}+i 0\right)^{-1} O p_{H}(a) h D_{\nu} \varphi_{h}\right|_{H},\left.h D_{\nu} \varphi_{h}\right|_{H}\right\rangle_{L^{2}(H)} \\
& \quad+\left\langle\left. O p_{H}(a) \varphi_{h}\right|_{H},\left.\varphi_{h}\right|_{H}\right\rangle_{L^{2}(H)} \\
& \rightarrow_{h \rightarrow 0^{+}} \frac{4}{\mu\left(S^{*} M\right)} \int_{B^{*} H} a_{0}\left(x^{\prime}, \xi^{\prime}\right)\left(1-\left|\xi^{\prime}\right|^{2}\right)^{-1 / 2} d \sigma
\end{aligned}
$$

where $a_{0}\left(x^{\prime}, \xi^{\prime}\right)$ is the principal symbol of $O p_{H}(a)$.

The additional step in the proof is a pointwise local Weyl law as in [TZ1] section 8.4 showing that only a sparse set of eigenfunctions could scar on the glancing set $S^{*} H$. This is precisely the step which is not allowed in the QUER problem. Therefore, QUER for all $O p_{H}(a)$ might fail for this rescaled problem; to determine whether it holds for all $O p_{H}(a)$ we would need a new idea. However, the following is a direct consequence of Theorem 2 .

Corollary 1.2. Suppose that $\left\{\varphi_{h}\right\}$ is $Q U E$ on $M$. Then the distributions $\left\{d \Phi_{h}^{D}+\right.$ $\left.d \Phi_{h}^{R N}\right\}$ have a unique weak* limit

$$
\omega(a):=\frac{4}{\mu\left(S^{*} M\right)} \int_{B^{*} H} a_{0}\left(x^{\prime}, \xi^{\prime}\right)\left(1-\left|\xi^{\prime}\right|^{2}\right)^{-1 / 2} d \sigma
$$

with respect to the subclass of symbols which vanish on $S^{*} H$.

We prove Theorem 1 by means of a Rellich identity adapted from [GL, Bu]. It is also possible to prove the theorem using the layer potential approach in Step 2 (Proof of (7.4)) in Section 7 of [HaZe]. To adapt this proof, one would need to introduce a semi-classical Green's function in place of the Euclidean Green's function, verify that it has the properties of the latter in section 4 of [HaZe], and then go through the proof of Step 2. Despite the authors' fondness for the layer potential approach, this proof is much longer than the infinitesimal Rellich identity approach and we have decided to omit the details.

\section{Rellich approach: Proof of Theorem 1}

We have assumed $H$ is a separating hypersurface, so that $H$ is the boundary of a smooth open submanifold of $M, H=\partial M_{+} \subset M$. There is no loss of generality in this assumption, since we may always use a cutoff to a subset of $H$. We then use a Rellich type identity to write the integral of a commutator over $M_{+}$as a sum of integrals over the boundary (of course the same argument would apply on $M_{-}=M \backslash M_{+}$). The argument is partially motivated by Burq's proof of boundary quantum ergodicity (ie. the case $H=\partial M)$.

Let $x=\left(x_{1}, \ldots, x_{n-1}, x_{n}\right)=\left(x^{\prime}, x_{n}\right)$ be Fermi normal coordinates in a small tubular neighbourhood $H(\epsilon)$ of $H$ defined near a point $x_{0} \in H$. In these coordinates, we can locally write

$$
H(\epsilon):=\left\{\left(x^{\prime}, x_{n}\right) \in U \times \mathbb{R},\left|x_{n}\right|<\epsilon\right\} .
$$

Here $U \subset \mathbb{R}^{n-1}$ is a coordinate chart containing $x_{0} \in H$ and $\epsilon>0$ is arbitrarily small but for the moment, fixed. We let $\chi \in C_{0}^{\infty}(\mathbb{R})$ be a cutoff with $\chi(x)=0$ for $|x| \geq 1$ 
and $\chi(x)=1$ for $|x| \leq 1 / 2$. In terms of the normal coordinates,

$$
-h^{2} \Delta_{g}=\frac{1}{g(x)} h D_{x_{n}} g(x) h D_{x_{n}}+R\left(x_{n}, x^{\prime}, h D_{x^{\prime}}\right)
$$

where, $R$ is a second-order $h$-differential operator along $H$ with coefficients that depend on $x_{n}$, and $R\left(0, x^{\prime}, h D_{x^{\prime}}\right)=-h^{2} \Delta_{H}$ is the induced tangential semiclassical Laplacian on $H$.

Let $A\left(x, h D_{x}\right) \in \Psi_{s c}^{0}(M)$ be an order zero semiclassical pseudodifferential operator on $M$ (see Appendix A). By Green's formula and (1.1) we get the Rellich identity

$$
\begin{aligned}
\frac{i}{h} \int_{M_{+}} & \left(\left[-h^{2} \Delta_{g}, A\left(x, h D_{x}\right)\right] \varphi_{h}(x)\right) \overline{\varphi_{h}(x)} d x \\
= & \left.\int_{H}\left(\left.h D_{\nu} A\left(x^{\prime}, x_{n}, h D_{x}\right) \varphi_{h}\right|_{H}\right) \overline{\varphi_{h}}\right|_{H} d \sigma_{H} \\
& +\left.\int_{H}\left(\left.A\left(x^{\prime}, x_{n}, h D_{x}\right) \varphi_{h}\right|_{H}\right) \overline{h D_{\nu} \varphi_{h}}\right|_{H} d \sigma_{H} .
\end{aligned}
$$

Here, $D_{x_{j}}=\frac{1}{i} \frac{\partial}{\partial x_{j}}, D_{x^{\prime}}=\left(D_{x_{1}}, \ldots, D_{x_{n-1}}\right), \quad D_{\nu}=\frac{1}{i} \partial_{\nu}$ where $\partial_{\nu}$ is the interior unit normal to $M_{+}$.

Given $a \in S^{0,0}\left(T^{*} H \times\left(0, h_{0}\right]\right)$, we then choose

$$
A\left(x^{\prime}, x_{n}, h D_{x}\right)=\chi\left(\frac{x_{n}}{\epsilon}\right) h D_{x_{n}} a^{w}\left(x^{\prime}, h D^{\prime}\right) .
$$

Since $\chi(0)=1$ it follows that the second term on the right-hand side of $(2.1)$ is just

$$
\left\langle\left. a^{w}\left(x^{\prime}, h D^{\prime}\right) h D_{x_{n}} \varphi_{h}\right|_{H},\left.h D_{x_{n}} \varphi_{h}\right|_{H}\right\rangle .
$$

The first term on right-hand side of (2.1) equals

$$
\begin{aligned}
& \left.\left.\int_{H} h D_{n}\left(\chi\left(x_{n} / \epsilon\right) h D_{n} a^{w}\left(x^{\prime}, h D^{\prime}\right) \varphi_{h}\right)\right|_{x_{n}=0} \overline{\varphi_{h}}\right|_{x_{n}=0} d \sigma_{H} \\
& =\int_{H}\left(\chi\left(x_{n} / \epsilon\right) a^{w}\left(x^{\prime}, h D^{\prime}\right)\left(h D_{n}\right)^{2} \varphi_{h}\right. \\
& \left.\quad \quad+\frac{h}{i \epsilon} \chi^{\prime}\left(x_{n} / \epsilon\right) h D_{n} a^{w}\left(x^{\prime}, h D^{\prime}\right) \varphi_{h}\right)\left.\left.\right|_{x_{n}=0} \overline{\varphi_{h}}\right|_{x_{n}=0} d \sigma_{H} \\
& =\left.\left.\int_{H}\left(\chi\left(x_{n} / \epsilon\right) a^{w}\left(x^{\prime}, h D^{\prime}\right)\left(1-R\left(x_{n}, x^{\prime}, h D^{\prime}\right)\right) \varphi_{h}\right)\right|_{x_{n}=0} \overline{\varphi_{h}}\right|_{x_{n}=0} d \sigma_{H}+\mathcal{O}_{\epsilon}(h),
\end{aligned}
$$

since $\chi^{\prime}(0)=0$ and $\left(\left(h D_{n}\right)^{2}+R+O(h)\right) \varphi_{h}=\varphi_{h}$ in these coordinates.

It follows from $(2.1)-(2.3)$ that

$$
\begin{aligned}
& \left\langle\left. O p_{H}(a) h D_{\nu} \varphi_{h}\right|_{H},\left.h D_{\nu} \varphi_{h}\right|_{H}\right\rangle_{L^{2}(H)}+\left\langle\left. O p_{H}(a)\left(1+h^{2} \Delta_{H}\right) \varphi_{h}\right|_{H},\left.\varphi_{h}\right|_{H}\right\rangle_{L^{2}(H)} \\
& =\left\langle\left(\left\{\xi_{n}^{2}+R\left(x_{n}, x^{\prime}, \xi^{\prime}\right), \chi\left(\frac{x_{n}}{\epsilon}\right) \xi_{n} a\left(x^{\prime}, \xi^{\prime}\right)\right\}\right)^{w} \varphi_{h}, \varphi_{h}\right\rangle_{L^{2}\left(M_{+}\right)}+\mathcal{O}_{\epsilon}(h)
\end{aligned}
$$

We now assume that $\varphi_{h}$ is a sequence of quantum ergodic eigenfunctions, and take the $h \rightarrow 0^{+}$limit on both sides of (2.4). We apply interior quantum ergodicity to the 
term on the right-hand side of (2.4). We compute

$$
\begin{aligned}
\left\{\xi_{n}^{2}+R\left(x_{n}, x^{\prime}, \xi^{\prime}\right), \chi\left(\frac{x_{n}}{\epsilon}\right) \xi_{n} a\left(x^{\prime}, \xi^{\prime}\right)\right\}= & \frac{2}{\epsilon} \chi^{\prime}\left(\frac{x_{n}}{\epsilon}\right) \xi_{n}^{2} a\left(x^{\prime}, \xi^{\prime}\right) \\
& +\chi\left(\frac{x_{n}}{\epsilon}\right) R_{2}\left(x^{\prime}, x_{n}, \xi^{\prime}\right)
\end{aligned}
$$

where $R_{2}$ is a zero-order symbol. Let $\chi_{2} \in \mathcal{C}^{\infty}$ satisfy $\chi_{2}(t)=0$ for $t \leq-1 / 2$, $\chi_{2}(t)=1$ for $t \geq 0$, and $\chi_{2}^{\prime}(t)>0$ for $-1 / 2<t<0$, and let $\rho$ be a boundary defining function for $M_{+}$. Then $\chi_{2}(\rho / \delta)$ is 1 on $M_{+}$and 0 outside a $\delta / 2$ neighbourhood. Now the assumptions that the sequence $\varphi_{h}$ is quantum ergodic implies that the matrix element of the second term on the right-hand side of (2.6) is bounded by

$$
\begin{aligned}
& \left|\left\langle\left(\chi\left(x_{n} / \epsilon\right) R_{2}\left(x, \xi^{\prime}\right)\right)^{w} \varphi_{h}, \varphi_{h}\right\rangle_{L^{2}\left(M_{+}\right)}\right| \\
& \quad \leq\left\|\chi_{2}(\rho / \delta) \chi\left(x_{n} / \epsilon\right) \varphi_{h}\right\|_{L^{2}(M)}\left\|\tilde{\chi}_{2}(\rho / \delta) \tilde{\chi}\left(x_{n} / \epsilon\right) \varphi_{h}\right\|_{L^{2}(M)} \\
& \quad=\mathcal{O}_{\delta}(\epsilon)+o_{\delta, \epsilon}(1)
\end{aligned}
$$

where $\tilde{\chi}$ and $\tilde{\chi}_{2}$ are smooth, compactly supported functions which are one on the support of $\chi$ and $\chi_{2}$ respectively. Here, the last line follows from interior quantum ergodicity of the $\varphi_{h}$ since the volume of the supports of $\chi\left(x_{n} / \epsilon\right)$ and $\tilde{\chi}\left(x_{n} / \epsilon\right)$ is comparable to $\epsilon$.

To handle the matrix element of the first term on the right-hand side of (2.6), we note that $\left.\chi^{\prime}\left(x_{n} / \epsilon\right)\right|_{M_{+}}=\tilde{\chi}^{\prime}\left(x_{n} / \epsilon\right)$ for a smooth function $\tilde{\chi} \in \mathcal{C}^{\infty}(M)$ satisfying $\tilde{\chi}=1$ in a neighbourhood of $M \backslash M_{+}$and zero inside a neighbourhood of $H$. Then, again by interior quantum ergodicity, we have

$$
\begin{aligned}
& 2\left\langle\left(\frac{1}{\epsilon} \chi^{\prime}\left(\frac{x_{n}}{\epsilon}\right) \xi_{n}^{2} a\left(x^{\prime}, \xi^{\prime}\right)\right)^{w} \varphi_{h}, \varphi_{h}\right\rangle_{L^{2}\left(M_{+}\right)} \\
& =2\left\langle\left(\frac{1}{\epsilon} \tilde{\chi}^{\prime}\left(\frac{x_{n}}{\epsilon}\right) \xi_{n}^{2} a\left(x^{\prime}, \xi^{\prime}\right)\right)^{w} \varphi_{h}, \varphi_{h}\right\rangle_{L^{2}(M)} \\
& =\frac{2}{\mu\left(S^{*} M\right)} \int_{S^{*} M} \frac{1}{\epsilon} \tilde{\chi}^{\prime}\left(\frac{x_{n}}{\epsilon}\right)\left(1-R\left(x^{\prime}, x_{n}, \xi^{\prime}\right)\right) a\left(x^{\prime}, \xi^{\prime}\right) d \mu+O(\epsilon)+o_{\epsilon}(1) \\
& =\frac{2}{\mu\left(S^{*} M\right)} \int_{S^{*} M_{+}} \frac{1}{\epsilon} \chi^{\prime}\left(\frac{x_{n}}{\epsilon}\right)\left(1-R\left(x^{\prime}, x_{n}, \xi^{\prime}\right)\right) a\left(x^{\prime}, \xi^{\prime}\right) d \mu+O(\epsilon)+o_{\epsilon}(1),
\end{aligned}
$$

since $\tilde{\chi}^{\prime}$ and $\chi^{\prime}$ are supported inside $M_{+}$. Combining the above calculations yields

$$
\begin{aligned}
& \left\langle\left. O p_{H}(a) h D_{\nu} \varphi_{h}\right|_{H},\left.h D_{\nu} \varphi_{h}\right|_{H}\right\rangle_{L^{2}(H)}+\left\langle\left. O p_{H}(a)\left(1+h^{2} \Delta_{H}\right) \varphi_{h}\right|_{H},\left.\varphi_{h}\right|_{H}\right\rangle_{L^{2}(H)} \\
& =\frac{2}{\mu\left(S^{*} M\right)} \int_{S^{*} M_{+}} \frac{1}{\epsilon} \chi^{\prime}\left(\frac{x_{n}}{\epsilon}\right)\left(1-R\left(x^{\prime}, x_{n}, \xi^{\prime}\right)\right) a\left(x^{\prime}, \xi^{\prime}\right) d \mu+O_{\delta}(\epsilon)+o_{\delta, \epsilon}(1) .
\end{aligned}
$$


Finally, we take the $h \rightarrow 0^{+}$-limit in (2.8) followed by the $\epsilon \rightarrow 0^{+}$-limit, and finally the $\delta \rightarrow 0^{+}$limit. The result is that, since the left-hand side in (2.8) is independent of $\epsilon$ and $\delta$,

$$
\begin{aligned}
\lim _{h \rightarrow 0^{+}} & \left\langle\left. O p_{H}(a) h D_{\nu} \varphi_{h}\right|_{H},\left.h D_{\nu} \varphi_{h}\right|_{H}\right\rangle_{L^{2}(H)}+\left\langle\left. O p_{H}(a)\left(1+h^{2} \Delta_{H}\right) \varphi_{h}\right|_{H},\left.\varphi_{h}\right|_{H}\right\rangle_{L^{2}(H)} \\
& =\frac{2}{\mu\left(S^{*} M\right)} \int_{S_{H}^{*} M}\left(1-R\left(x^{\prime}, x_{n}=0, \xi^{\prime}\right)\right) d \tilde{\sigma} \\
& \left.=\frac{4}{\mu\left(S^{*} M\right)} \int_{B^{*} H}\left(1-\left|\xi^{\prime}\right|^{2}\right)^{1 / 2} a\left(x^{\prime}, \xi^{\prime}\right)\right) d \sigma,
\end{aligned}
$$

where $d \tilde{\sigma}$ is the symplectic volume form on $S_{H}^{*} M$, and $d \sigma$ is the symplectic volume form on $B^{*} H$.

\section{Proof of Theorem 2 and Corollary 1.2}

The proof follows as in Theorem 1 with a few modifications. For fixed $\epsilon_{1}>0$ we choose the test operator

$$
A\left(x^{\prime}, x_{n}, h D_{x}\right):=\left(I+h^{2} \Delta_{H}\left(x^{\prime}, h D^{\prime}\right)+i \epsilon_{1}\right)^{-1} \chi\left(\frac{x_{n}}{\epsilon}\right) h D_{x_{n}} a^{w}\left(x^{\prime}, h D^{\prime}\right)
$$

and since $W F_{h}^{\prime}\left(\left.\varphi_{h}\right|_{H}\right) \subset B^{*} H$ (see [TZ2], Section 11) it suffices to assume that $a \in C_{0}^{\infty}\left(T^{*} H\right)$ with

$$
\operatorname{supp} a \subset B_{1+\epsilon_{1}^{2}}^{*}(H) \text {. }
$$

Let $\chi_{\epsilon_{1}}\left(x^{\prime}, \xi^{\prime}\right) \in C_{0}^{\infty}\left(B_{1+\epsilon_{1}^{2}}^{*}(H) \backslash B_{1-2 \epsilon_{1}^{2}}^{*}(H) ;[0,1]\right)$ be a cutoff near the glancing set $S^{*} H$ with $\chi_{\epsilon_{1}}\left(x^{\prime}, \xi^{\prime}\right)=1$ when $\left(x^{\prime}, \xi^{\prime}\right) \in B_{1+\epsilon_{1}^{2}}^{*}(H) \backslash B_{1-\epsilon_{1}^{2}}^{*}(H)$. Then, with $A\left(x, h D_{x}\right)$ in (3.1), the same Rellich commutator argument as in Theorem 1 gives

$$
\begin{aligned}
& \left\langle\left.\left(1+h^{2} \Delta_{H}+i \epsilon_{1}\right)^{-1} a^{w}\left(x^{\prime}, h D^{\prime}\right)\left(1-\chi_{\epsilon_{1}}\right)^{w} h D_{\nu} \varphi_{h}\right|_{H},\left.h D_{\nu} \varphi_{h}\right|_{H}\right\rangle_{L^{2}(H)} \\
& \quad+\left\langle\left. a^{w}\left(x^{\prime}, h D^{\prime}\right)\left(1-\chi_{\epsilon_{1}}\right)^{w}\left(\frac{1-\left|\xi^{\prime}\right|^{2}}{1-\left|\xi^{\prime}\right|^{2}+i \epsilon_{1}}\right)^{w} \varphi_{h}\right|_{H},\left.\varphi_{h}\right|_{H}\right\rangle_{L^{2}(H)} \\
& \rightarrow \frac{4}{\mu\left(S^{*} M\right)} \int_{B^{*} H} a_{0}\left(x^{\prime}, \xi^{\prime}\right)\left(1-\chi_{\epsilon_{1}}\left(x^{\prime}, \xi^{\prime}\right)\right)\left(\frac{\left(1-\left|\xi^{\prime}\right|^{2}\right)^{1 / 2}}{1-\left|\xi^{\prime}\right|^{2}+i \epsilon_{1}}\right) d \sigma .
\end{aligned}
$$

It remains to determine the contribution of the glancing set $S^{*} H$. As in [Bu,DZ,HaZe, TZ1] we use a local Weyl law to do this. Because of the additional normal derivative term the argument is slightly different than in the cited articles and so we give some details. For the rest of this proof, we need to recall that $h \in\left\{\lambda_{j}^{-1}\right\}$, and we write $h_{j}$ for this sequence to emphasize that it is a discrete sequence of values $h_{j} \rightarrow 0$. Since $\left\|a^{w}\left(x^{\prime}, h D^{\prime}\right)\right\|_{L^{2} \rightarrow L^{2}}=O(1)$, it follows that for $h \in\left(0, h_{0}\left(\epsilon_{1}\right)\right]$ with $h_{0}\left(\epsilon_{1}\right)>0$ 
sufficiently small,

$$
\begin{aligned}
& \frac{1}{N(h)} \sum_{h_{j} \geq h}\left|\left\langle\left. a^{w}\left(x^{\prime}, h D^{\prime}\right) \chi_{\epsilon_{1}}^{w} \varphi_{h_{j}}\right|_{H}, \varphi_{h_{j}} \mid H\right\rangle_{L^{2}(H)}\right| \\
& \quad \leq C \frac{1}{N(h)} \sum_{h_{j} \geq h}\left(\left\|\left.\chi_{\epsilon_{1}}^{w} \varphi_{h_{j}}\right|_{H}\right\|_{L^{2}(H)}\left\|\left.\chi_{2 \epsilon_{1}}^{w} \varphi_{h_{j}}\right|_{H}\right\|_{L^{2}(H)}+\mathcal{O}\left(h_{j}^{\infty}\right)\right) \\
& \quad \leq \frac{C}{2} \frac{1}{N(h)} \sum_{h_{j} \geq h}\left(\left\|\left.\chi_{\epsilon_{1}}^{w} \varphi_{h_{j}}\right|_{H}\right\|_{L^{2}(H)}^{2}+\left\|\left.\chi_{2 \epsilon_{1}}^{w} \varphi_{h_{j}}\right|_{H}\right\|_{L^{2}(H)}^{2}+\mathcal{O}\left(h_{j}^{\infty}\right)\right) \\
& \quad=\mathcal{O}\left(\epsilon_{1}^{2}\right) .
\end{aligned}
$$

By a Fourier Tauberian argument [TZ1] in Section 8.4, it follows that for $h \in$ $\left(0, h_{0}\left(\epsilon_{1}\right)\right]$

$$
\left.\frac{1}{N(h)} \sum_{h_{j} \geq h}\left|\chi_{\epsilon_{1}, 2 \epsilon_{1}}^{w} \varphi_{h_{j}}\right|_{H}\left(x^{\prime}\right)\right|^{2}=\mathcal{O}\left(\epsilon_{1}^{2}\right)
$$

uniformly for $x^{\prime} \in H$. The last estimate in (3.3) follows from (3.4) by integration over $H$.

To estimate the normal derivative terms, we first recall the standard resolvent estimate

$$
\left\|\left(1+h^{2} \Delta_{H}+i \epsilon_{1}\right)^{-1} u\right\|_{H_{h}^{2}(H)} \leq C \epsilon_{1}^{-1}\|u\|_{L^{2}(H)},
$$

where $H_{h}^{2}$ is the semiclassical Sobolev space of order 2 (see [Zw] Lemma 13.6). Applying the obvious embedding $H_{h}^{2}(H) \subset L^{2}(H)$, we recover

$$
\begin{aligned}
\left\|\left(1+h^{2} \Delta_{H}+i \epsilon_{1}\right)^{-1} u\right\|_{L^{2}(H)} & \leq C\left\|\left(1+h^{2} \Delta_{H}+i \epsilon_{1}\right)^{-1} u\right\|_{H_{h}^{2}(H)} \\
& \leq C \epsilon_{1}^{-1}\|u\|_{L^{2}(H)}
\end{aligned}
$$

to get that

$$
\begin{aligned}
& \frac{1}{N(h)} \sum_{h_{j} \geq h}\left|\left\langle\left.\left(1+h^{2} \Delta_{H}+i \epsilon_{1}\right)^{-1} a^{w}\left(x^{\prime}, h D^{\prime}\right) \chi_{\epsilon_{1}}^{w} h_{j} D_{x_{n}} \varphi_{h_{j}}\right|_{H},\left.h_{j} D_{x_{n}} \varphi_{h_{j}}\right|_{H}\right\rangle_{L^{2}(H)}\right| \\
& \quad \leq C^{\prime} \epsilon_{1}^{-1} \frac{1}{N(h)} \sum_{h_{j} \geq h}\left(\left\|\left.\chi_{\epsilon_{1}}^{w} h D_{x_{n}} \varphi_{h_{j}}\right|_{H}\right\|_{L^{2}(H)}\left\|\left.\chi_{2 \epsilon_{1}}^{w} h D_{x_{n}} \varphi_{h_{j}}\right|_{H}\right\|_{L^{2}(H)}+\mathcal{O}\left(h_{j}^{\infty}\right)\right) \\
& \quad \leq \frac{C^{\prime} \epsilon_{1}^{-1}}{2} \frac{1}{N(h)} \sum_{h_{j} \geq h}\left(\left\|\left.\chi_{\epsilon_{1}}^{w} h D_{x_{n}} \varphi_{h_{j}}\right|_{H}\right\|_{L^{2}(H)}^{2}+\left\|\left.\chi_{2 \epsilon_{1}}^{w} h D_{x_{n}} \varphi_{h_{j}}\right|_{H}\right\|_{L^{2}(H)}^{2}+\mathcal{O}\left(h_{j}^{\infty}\right)\right) \\
& \quad=\mathcal{O}\left(\epsilon_{1}^{-1} \epsilon_{1}^{2}\right) \\
& \quad=\mathcal{O}\left(\epsilon_{1}\right) .
\end{aligned}
$$

The last estimate follows again from the Fourier Tauberian argument in [TZ1] section 8.4, which gives

$$
\left.\frac{1}{N(h)} \sum_{h_{j} \geq h}\left|\chi_{\epsilon_{1}, 2 \epsilon_{1}}^{w} h_{j} D_{x_{n}} \varphi_{h_{j}}\right|_{H}\left(x^{\prime}\right)\right|^{2}=\mathcal{O}\left(\epsilon_{1}^{2}\right)
$$

uniformly for $x^{\prime} \in H$. 
Since $\epsilon_{1}>0$ is arbitrary, Theorem 2 follows from (3.3) and (3.5) by letting $\epsilon_{1} \rightarrow 0^{+}$ in $(3.2)$.

3.1. Proof of Corollary 1.2. We now observe that Corollary 1.2 follows almost immediately from the proof of Theorem 2 . To see this, we note that by restricting our attention to symbols which vanish on the glancing set, we do not need to pass through the local Weyl law/Tauberian argument, which is the step by which one extracts a density one subsequence. Hence the result applies to the full sequence.

\section{Appendix A}

A.1. Semiclassical symbols. Let $M$ be a compact manifold. By a semiclassical symbol $a \in S^{m, k}\left(T^{*} M \times\left[0, h_{0}\right)\right)$, we mean a smooth function possessing an asymptotic expansion as $h \rightarrow 0$ of the form,

$$
a(x, \xi, h) \sim_{h \rightarrow 0^{+}} \sum_{j=0}^{\infty} a_{k-j}(x, \xi) h^{m+j},
$$

with $a_{k-j} \in S_{1,0}^{k}\left(T^{*} M\right)$. Here, $S_{1,0}^{k}$ is the standard Hörmander class consisting of smooth functions $a(x, \xi)$ satisfying the estimates $\left|\partial_{x}^{\alpha} \partial_{\xi}^{\beta} a(x, \xi)\right| \leq C_{\alpha, \beta}\langle\xi\rangle^{k-|\beta|}$ for all multi-indices $\alpha, \beta \in N^{n}$. We say that $A(h) \in O p_{h}^{w}\left(S^{m, k}\left(T^{*} M \times\left[0, h_{0}\right)\right)\right.$ provided its Schwartz kernel is locally of the form

$$
A(h)(x, y)=(2 \pi h)^{-n} \int_{\mathbb{R}^{n}} e^{i\langle x-y, \xi\rangle / h} a\left(\frac{x+y}{2}, \xi, h\right) d \xi
$$

with $a \in S^{m, k}$. We denote the operator $A(h)$ by $a^{w}\left(x, h D_{x}\right)$ (or simply $a^{w}$ ). By a symbol of order zero we mean that $a \in S^{0,0}$, and we refer to $a_{0}(x, \xi)$ as the principal symbol. In the latter case, we simply write

$$
S_{s c}^{0}(M):=S^{0,0}, \quad \Psi_{s c}^{0}(M):=O p_{h}^{w}\left(S^{0,0}\right) .
$$

Finally, when $H \subset M$ is a hypersurface and $a \in S_{s c}^{0}(H)$, we sometimes write $O p_{H}(a)=a^{w}$ to indicate dependence on the submanifold, $H$. We refer to [Zw] for background.

\section{Acknowledgments}

The first version of this paper was written at the same time as [TZ1,TZ2] but its completion was postponed while the authors proved the QER phenomenon for Dirichlet data alone. We were further stimulated to complete the article by a discussion with Peter Sarnak at the Spectral Geometry conference at Dartmouth in July, 2010 in which we debated whether QUE in the ambient domain implies QUER along $H$. We said "yes", Sarnak said "no"; Corollaries 1.1 and 1.2 explain the sense in which both answers are right.

The research of H.C. was partially supported by NSF grant no. DMS-0900524; J.T. was partially supported by NSERC grant no. OGP0170280 and a William Dawson Fellowship; S.Z. was partially supported by NSF grant no. DMS-0904252. 


\section{References}

[Bu] N. Burq, Quantum ergodicity of boundary values of eigenfunctions: a control theory approach, Canad. Math. Bull. 48(1) (2005), 3-15.

[CdV] Y. Colin de Verdière, Ergodicité et fonctions propres du laplacien, Commun. Math. Phys. 102 (1985), 497-502.

[DZ] S. Dyatlov and M. Zworski, Quantum ergodicity for restrictions to hypersurfaces, Nonlinearity 26 (2013), 35-52. (arXiv:1204.0284).

[GL] P. Gérard and E. Leichtnam, Ergodic properties of eigenfunctions for the Dirichlet problem, Duke Math. J. 71(2) (1993), 559-607.

[HaZe] A. Hassell and S. Zelditch, Quantum ergodicity of boundary values of eigenfunctions, Commun. Math. Phys. 248(1) (2004), 119-168.

[Sch] A.I. Schnirelman, Ergodic properties of eigenfunctions, Usp. Math. Nauk. 29 (1974), 181-182.

[TZ1] J.A. Toth and S. Zelditch, Quantum ergodic restriction theorems, I: interior hypersurfaces in domains with ergodic billiards, Ann. Henri Poincaré 13(4) (2012), 599-670; arXiv: 1005.1636.

[TZ2] J.A. Toth and S. Zelditch, Quantum ergodic restriction theorems: manifolds without boundary, Geom. Func. Anal. 23 (2013), 715-775.

[Zel] S. Zelditch, Uniform Distribution of Eigenfunctions on Compacty Hyperbolic Surfaces, Duke Math. J. 55 (1987), 919-941.

[ZZ] S. Zelditch and M. Zworski, Ergodicity of eigenfunctions for ergodic billiards, Commun. Math. Phys. 175 (1996), 673-682.

[Zw] M. Zworski, Semiclassical Analysis, Graduate Studies in Mathematics 138, AMS, 2012.

Department of Mathematics, UnC-Chapel Hill, CB\#3250 Phillips Hall, Chapel Hill, NC 27599, USA

E-mail address: hans@math.unc.edu

Department of Mathematics and Statistics, McGill University, Montreal, Canada

E-mail address: jtoth@math.mcgill.ca

Department of Mathematics, Northwestern University, Evanston, IL 60208-2370, USA

E-mail address: zelditch@math.northwestern.edu 
